\title{
Read-out Electronics and Signal Processing in GERDA and Future Prospects
}

\author{
Valerio D'Andrea* on behalf of the GERDA Collaboration \\ *Dipartimento di Scienze Fisiche e Chimiche, Università degli Studi dell'Aquila \\ \& INFN Laboratori Nazionali del Gran Sasso, Assergi, Italy \\ Email: valerio.dandrea@lngs.infn.it
}

\begin{abstract}
The GERDA experiment searches for the neutrinoless double beta decay of ${ }^{76} \mathrm{Ge}$. The experiment is using $36 \mathrm{~kg}$ of high-purity germanium detectors, simultaneously as source and detector, deployed into ultra-pure cryogenic liquid argon. GERDA is one the leading experiment in the field, reporting the highest sensitivity on the half-life of $0 \nu \beta \beta$ decay with $1.1 \cdot 10^{26} \mathbf{y r}$, the lowest background index with $6 \cdot 10^{-4} \mathrm{cts} /(\mathrm{keV} \cdot \mathrm{kg} \cdot \mathrm{yr})$ and an excellent energy resolution of $0.12 \%$ (FWHM).

The search for the $0 \nu \beta \beta$ decay of the isotope ${ }^{76} \mathrm{Ge}$ will be continued in the next years by the LEGEND-200 experiment, that aims to reach a sensitivity up to $10^{27} \mathrm{yr}$ using $200 \mathrm{~kg}$ of enriched HPGe detectors. The preparation of this experiment already started.

The basic concepts of the GERDA read-out electronics, obeying both the severe requirements of ultra high radio-purity and cryogenic operation, are summarized. For LEGEND-200 a new electronics design, including a separation of the preamplifier in two stages, has been already designed and realized: results from tests are presented.

Additionally, we will introduce the digital signal processing adopted for the energy reconstruction in GERDA and a new implementation of an optimum digital filter by means of the DPLMS method. This method are discussed and the first application to GERDA data are presented.
\end{abstract}

\section{GERDA EXPERIMENT}

The GErmanium Detector Array (GERDA) experiment [1] is searching for neutrinoless double beta $(0 \nu \beta \beta)$ decay of the isotope ${ }^{76} \mathrm{Ge}$. It is located at the underground Laboratori Nazionali del Gran Sasso (LNGS) of INFN in Italy. A rock overburden of about $3500 \mathrm{~m}$ water equivalent removes the hadronic components of cosmic ray showers and reduces the muon flux at the experiment.

The GERDA setup, illustrated in Fig. 1, has been designed following a multi-layer approach. High-Purity Germanium (HPGe) detectors enriched to about $87 \%$ in ${ }^{76} \mathrm{Ge}$ are operated bare in liquid argon (LAr), being both source and detector of $0 \nu \beta \beta$ decay. The LAr cryostat is complemented by a water tank with $10 \mathrm{~m}$ diameter which further shields from neutron and $\gamma$ backgrounds and also works as muon veto.

After a first physics data taking campaign [2] carried out from 2011 to 2013, the GERDA setup has been upgraded to perform the next step [3]. The major upgrade is the introduction of 30 new BEGe detectors from Canberra [4] with an optimal energy resolution, due to the low input capacitance $(\sim \mathrm{pF})$, and a powerful pulse shape discrimination (PSD), thanks to the configuration of the $\mathrm{p}^{+}$and $\mathrm{n}^{+}$contacts that produce a highly non-uniform electrical field. In addition, an

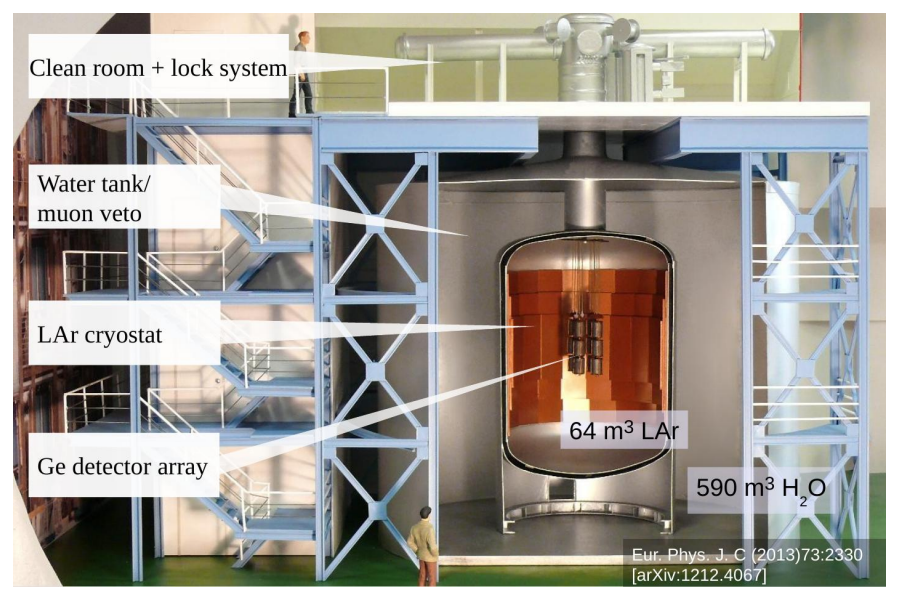

Fig. 1. GERDA schematic view [1].

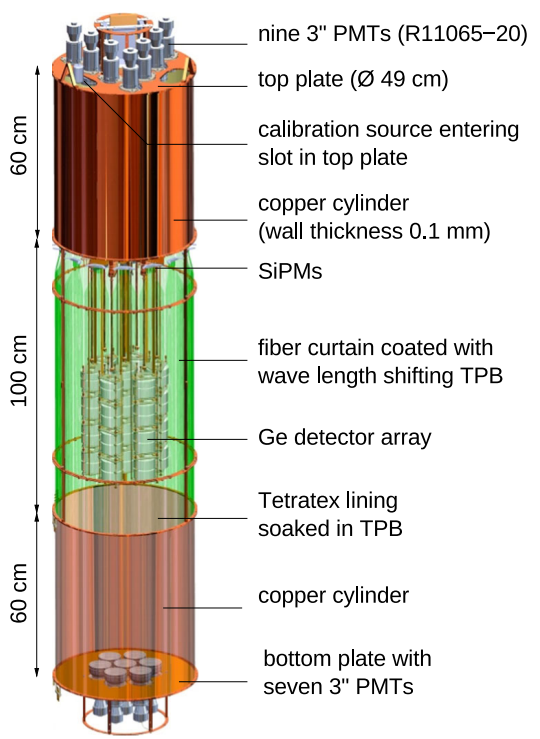

Fig. 2. Assembly of detector array and LAr veto system [3].

active suppression of the background by detecting the LAr scintillation light, using PMTs and wavelength shifting fibers coupled to SiPMs, has been introduced [5]. The core of the GERDA setup is shown in Fig. 2: the Ge detector array (30 BEGe, 7 enriched coaxial and 3 natural coaxial detectors) is at the center of the instrumented LAr volume. 
Initial results from the second phase, with about $10 \mathrm{~kg} \cdot \mathrm{yr}$ exposure, indicate that the target background of $10^{-3} \mathrm{cts} /(\mathrm{keV} \cdot \mathrm{kg} \cdot \mathrm{yr})$ is achieved, thus making GERDA the first experiment in the field which will be "background-free" up to the design exposure of $100 \mathrm{~kg} \cdot \mathrm{yr}$ [6]. This condition has been confirmed also in the following data release [7].

After more than two years of data taking after the upgrade, the total available enriched $\mathrm{Ge}$ exposure at the last data release of June 2018 is $82.4 \mathrm{~kg} \cdot \mathrm{yr}$ [8]. The $0 \nu \beta \beta$ decay analysis yielded no signal, setting a new limit on the ${ }^{76} \mathrm{Ge} 0 \nu \beta \beta$ decay half-life of $T_{1 / 2}^{0 \nu}>0.9 \cdot 10^{26} \mathrm{yr}(90 \%$ C.L.) with a median sensitivity of $1.1 \cdot 10^{26} \mathrm{yr}(90 \%$ C.L.), thus making GERDA the first $0 \nu \beta \beta$ decay experiment to surpass $10^{26} \mathrm{yr}$ sensitivity. The probability to obtain a limit stronger than the actual one in an ensemble of repeated experiments with null signal is $63 \%$.

\section{LEGEND EXPERIMENT}

Based on the success of GERDA and MAJORANA [9], the search for $0 \nu \beta \beta$ decay in ${ }^{76} \mathrm{Ge}$ will be continued in the next years by LEGEND [10] (Large Enriched Germanium Experiment for Neutrinoless $\beta \beta$ Decay). LEGEND will proceed in phases towards a $0 \nu \beta \beta$ decay discovery potential at a half-life beyond $10^{28}$ yr. The best technologies will be selected based on lessons learned in GERDA and MAJORANA, as well as contributions from other groups.

In its first phase, LEGEND-200, the existing GERDA infrastructure at LNGS will be modified to deploy $200 \mathrm{~kg}$ of detectors in the cryostat. LEGEND-200 has a background goal of less than $0.6 \mathrm{cts} /(\mathrm{FWHM} \cdot \mathrm{ton} \cdot \mathrm{yr})$. Achieving this background rate will allow to reach a sensitivity greater than $10^{27} \mathrm{yr}$ with 1 ton $\cdot y r$ of exposure.

Multiple techniques are already planned to achieve the required background reduction, such as the use of the MAJORANA electroformed copper, the upgrade of the GERDA liquid argon veto and the improvement of the front-end electronics, described in next sections. A crucial point is the choice of a new detector geometry, the Inverted Coaxial Point Contact (ICPC) detector [11], with similar performance to the BEGe and PPC detectors and a mass as large as a coaxial detector. Five enriched ICPC detectors have been already produced and deployed in GERDA in May 2018.

The second stage of LEGEND will occur in a new infrastructure, with $1000 \mathrm{~kg}$ of detectors deployed. The background goal for LEGEND-1000 is less than $0.1 \mathrm{cts} /(\mathrm{FWHM} \cdot \mathrm{ton} \cdot \mathrm{yr}$ ). This background reduction is necessary to achieve a $0 \nu \beta \beta$ decay discovery potential at a half-life greater than $10^{28} \mathrm{yr}$ on a reasonable timescale. The required depth to keep cosmogenic activation backgrounds (e.g. ${ }^{77 m} \mathrm{Ge}$ ) within the background budget is currently under investigation and will be a contributing factor in the choice of site.

\section{READ-OUT ELECTRONICS IN GERDA}

The peculiar GERDA situation, with Ge detector working directly in LAr, and the requirement of very low radioactive background, introduces several constraints on the design of the Ge detector readout.

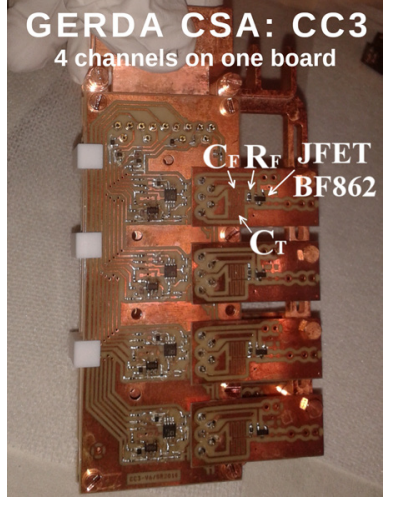

Fig. 3. CC3: GERDA read-out electronics.

The front-end electronics selected for GERDA is the CC3 [12], a hybrid and cryogenic Charge Sensitive Amplifier (CSA), designed to preserve the energy resolution of $\mathrm{Ge}$ detectors and to provide higher bandwidth for PSD. A picture of the CC3 board, placing 4 channels, is shown in Fig. 3.

The CC3 is manufactured with highly radio-pure materials on a $0.8 \mathrm{~mm}$ thick Cuflon substrate with two low-noise amplifiers with CMOS and Si Bipolar technology and the very front-end (VFE) section with the JFET BF862 from NXP Semiconductors and the feedback components: a resistor of $500 \mathrm{M} \Omega$ and a capacitor of $0.4 \mathrm{pF}$.

During GERDA data taking a performance degradation related to the Ge detector position in the strings has been observed [13]. In best cases an energy resolution of $0.1 \%$ (FWHM at $2.6 \mathrm{MeV}$ ) is observed in BEGe detectors, in agreement with previous results. Going down in the string the resolution deteriorate until $0.15 \%$. A suitable explanation for this behavior is that the signal cable (from detector to CC3) introduces a stray capacitance, increasing the noise and reducing energy resolution and PSD power. This observation motivated to put effort in the realization of a new read-out electronics for the LEGEND-200 experiment, including an amplifier stage close to each detector.

\section{NEW READ-OUt ELECTRONICS FOR LEGEND-200}

A new read-out with the CSA separated in 2 stages, has been already designed, realized and tested. The first stage, namely the Low Mass Front End (LMFE), to be placed in the vicinity of the detector, and the second stage, called $\mathrm{CC} 4$, to be located $40-50 \mathrm{~cm}$ far from detector array.

The LMFE has been designed and assembled in the Lawrence Berkeley National Laboratory, a picture is shown in Fig. 4. It is realized with ultra-pure materials to fit the stringent condition of LEGEND-200: the circuit is made on a Suprasil substrate, a high purity synthetic fused silica material, with sputtered Ti/Au traces. The LMFE is placing a bare die JFET (two options are tested: the Moxtek MX11 and the SF291 from Semefab), the feedback resistor (in amorphous germanium), feedback and test capacitors (made between traces).

The second stage of the new read-out has been designed and assembled in Milano and is based on GERDA CSA with two 


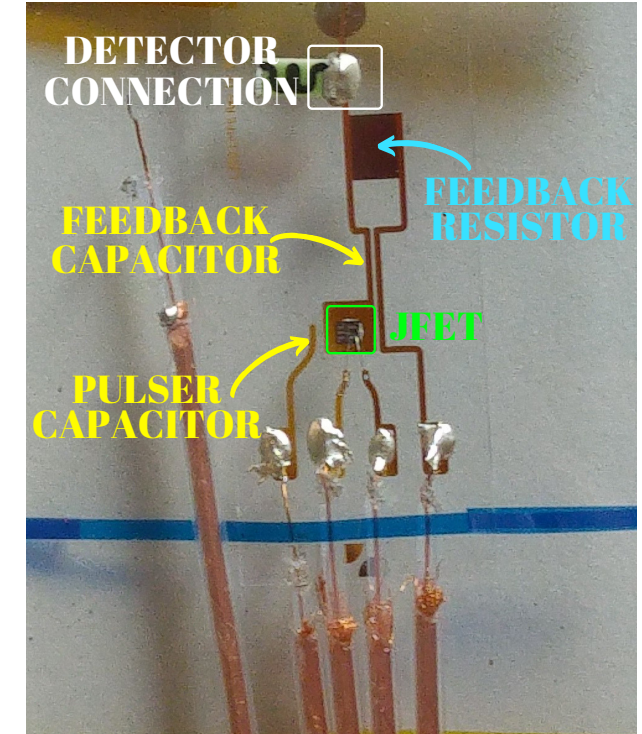

Fig. 4. Low Mass Front End (LMFE) realized in the Lawrence Berkeley National Laboratory [14].

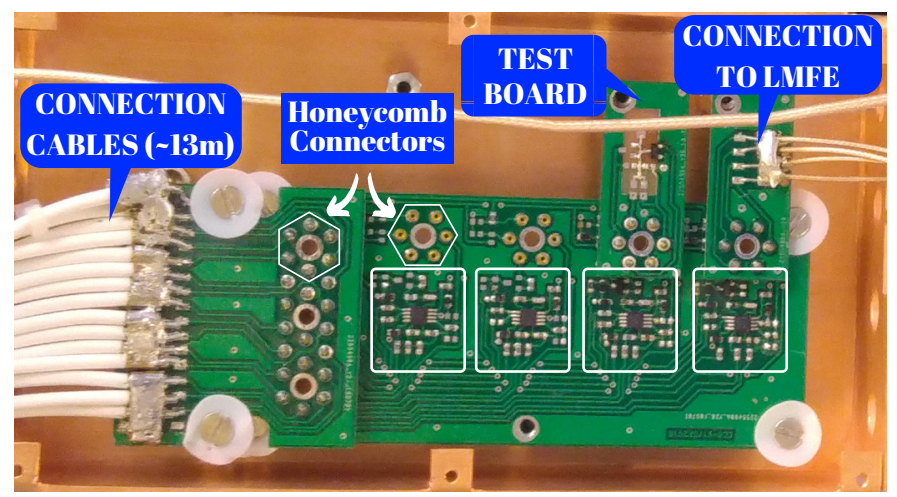

Fig. 5. Prototype of the CC4 (in this version the LMH6655 operational amplifier from Texas Instruments is used).

LMH6654 operational amplifier from Texas Instruments and the addition of a differential output to increase the dynamic range. The picture of the prototype is shown in Fig. 5: connectors with "honeycomb" shape are introduced in order to increase the mechanical stability of the system. The final version will be realized on a Cuflon or Kapton substrate.

First tests of the integration between LMFE and CC4 reported an energy resolution with a test pulse signal of $0.7 \mathrm{keV}$ (FWHM), a dynamic range larger than $9 \mathrm{MeV}$ with a $0.48 \mathrm{pF}$ feedback capacitor, a signal rise time of $\lesssim 50 \mathrm{~ns}$ with $3 \mathrm{pF}$ of input capacitor and a power consumption of $\sim 200 \mathrm{~mW} / \mathrm{chn}$.

\section{Signal Processing in Gerda}

It has been observed that GERDA detectors are sometimes affected by low frequency noise because of detector to frontend distance, microphonism induced by liquid argon and picked-up $50 \mathrm{~Hz}$ disturbances. In order to preserve their

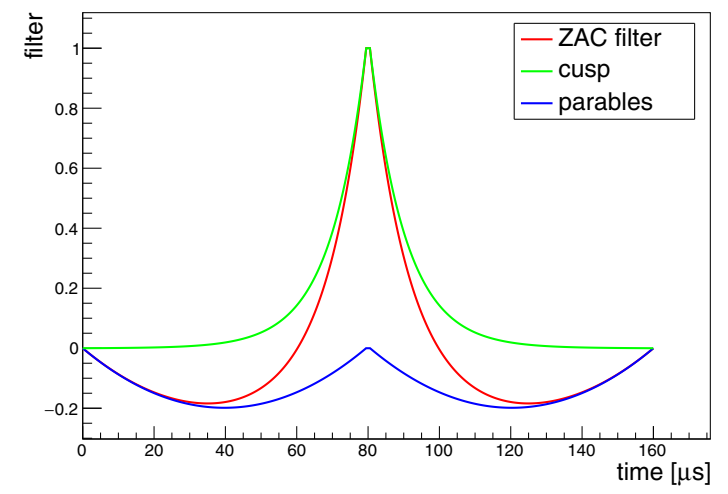

Fig. 6. ZAC filter: finite-length cusp (in green) and two negative parabolas (in blue) are summed to obtain the ZAC filter (in red) [13].

intrinsic energy resolution and PSD capability, a non-standard digital processing technique had to be developed.

From theory it can be shown that in case of series and parallel noise, the optimum shaping filter for energy estimation is an infinite cusp. Then, using a filter with total area equal to zero the low-frequency noise can be removed. In addition, the low-frequency baseline fluctuations are well subtracted by filters with parabolic shape.

Following these arguments a good shaping filter for GERDA is a cusp-like filter with zero total area, obtained by subtracting two parabolas. Also, to allow the full charge collection of the detector a flat-top must be added. The resulting filter is a ZeroArea finite-length Cusp-like (ZAC) filter with central flat-top [15], currently used for energy estimation in GERDA [13]. Fig. 6 shows an example of the ZAC filter construction: a cusp-like filter (in green) and two negative parabolas (in blue) are summed up to obtain the final filter (in red).

This filter improves the energy resolution with respect to a standard gaussian filter. The FWHM at $Q_{\beta \beta}=2039 \mathrm{keV}$ obtained in GERDA is 3.0 (3.6) $\mathrm{keV}$ for BEGe (coaxial) detectors.

However, the ZAC filter is not able to treat sinusoidal disturbances which are also observed in GERDA detectors, as shown in the FFT power spectrum of Fig. 7 obtained with a BEGe detector. These disturbances reduce the effectiveness of the ZAC filter.

\section{IMPLEMENTATION OF THE DPLMS FILTER}

An optimum filter, based on the individual detector, is needed in GERDA in order to treat a general noise situation and remove sinusoidal and other disturbances. In addition, the use of an optimum filter is more suitable with a high number of detectors (e.g. LEGEND) since the filter is adapted to the specific noise situation and no further adjustment is needed.

An optimum filter suitable for the GERDA situation is the Digital Penalized Least Mean Square (DPLMS) filter [16]. The method is based on the minimization of a figure of merit composed of experimental noise variance at the filter output and additional weighted constraints that impose some 


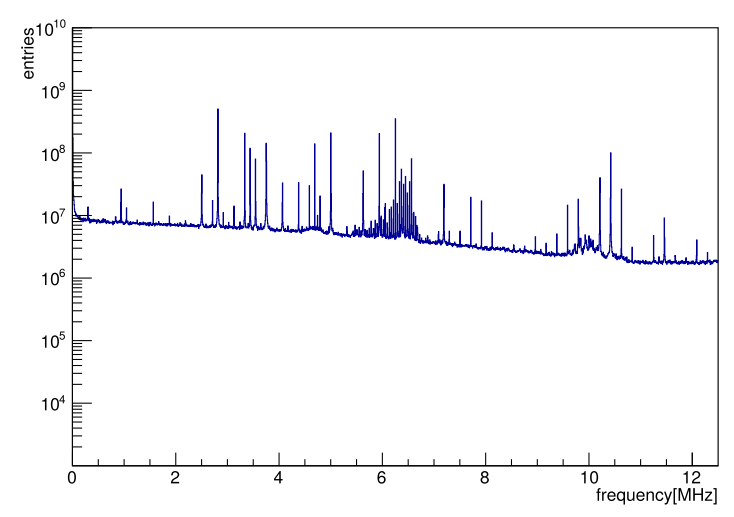

Fig. 7. FFT power spectrum obtained in GERDA with a BEGe detector.
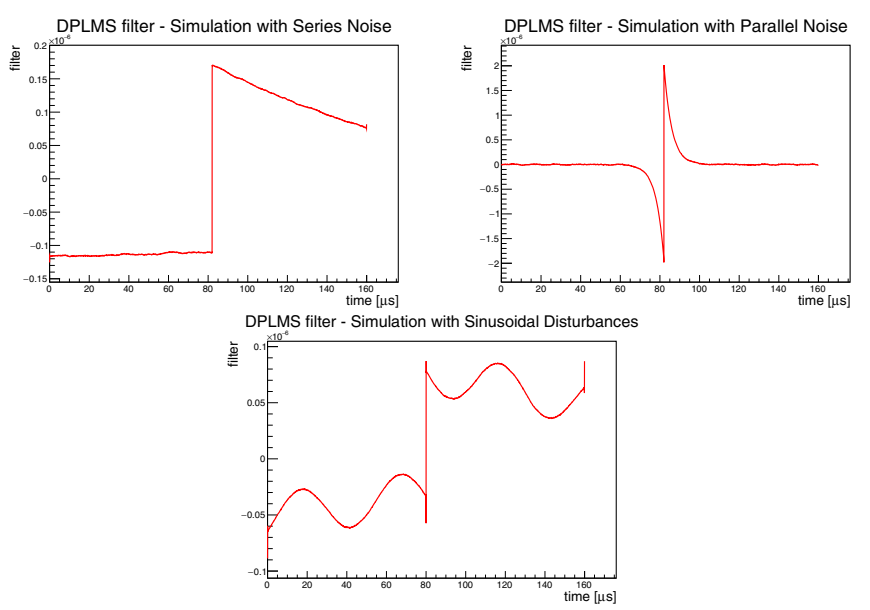

Fig. 8. DPLMS filter obtained with different simulated signals as indicated in the top legend.

appropriate deterministic behavior: pile-up removal, addition of a proper flat-top and zero-area condition. This filter works directly in the time domain, no deconvolution from frequency domain is needed (as the case of other optimum filters).

A C++ based software has been developed to create DPLMS filters starting from experimental signals.

\section{A. DPLMS filter with simulated waveforms}

Simulated waveforms that reproduce a signal coming from a GERDA detector have been used to characterize the DPLMS filter. Different types of experimental situations can be added to signals: series and parallel noise, sinusoidal disturbances at various frequencies, baseline movements and pile-up. Few examples of the DPLMS filter produced in different simulations are reported in Fig. 8: the case of only series noise (left upper panel), with the addition of parallel noise (right upper panel) and with a sinusoidal disturbance at frequency of $20 \mathrm{kHz}$ (lower panel).

The resolutions obtained with the reconstruction using the DPLMS filter have been compared with standard gaussian and ZAC filters. As shown in Fig. 9, with increasing series and parallel noise, the DPLMS filter gives a larger improvement with respect to other filters.
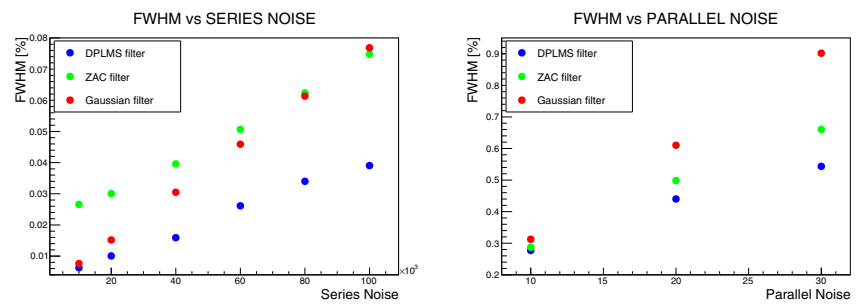

Fig. 9. Comparison of resolutions (in FWHM) obtained using DPLMS (in blue), ZAC (in green) and gaussian (in red) filters as function of the series (left panel) and parallel (right panel) noises.
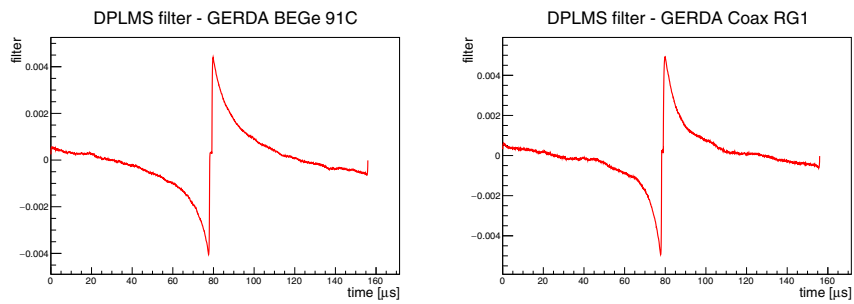

Fig. 10. DPLMS filters obtained in GERDA with a BEGe (left) and a coaxial (right) detector.

TABLE I

COMPARISON OF FWHM AT THE 2.6 MEV PEAK OBTAINED WITH GAUSSIAN, ZAC AND DPLMS FILTERS IN THREE GERDA DETECTORS.

\begin{tabular}{c|c|c|c} 
detector & Gaussian & ZAC filter & DPLMS filter \\
\hline BEGe-91A & $2.77 \pm 0.06$ & $2.67 \pm 0.06$ & $2.61 \pm 0.05$ \\
BEGe-91C & $3.52 \pm 0.06$ & $3.38 \pm 0.06$ & $3.24 \pm 0.05$ \\
Coax-RG1 & $4.77 \pm 0.04$ & $4.34 \pm 0.04$ & $4.22 \pm 0.03$
\end{tabular}

Sinusoidal disturbances are well removed by the DPLMS filter, while other filters are not able to treat this situation and very bad resolution is achieved.

\section{B. DPLMS filter with GERDA data}

In a second step, the DPLMS filter method has been applied to signals coming from GERDA detectors. Three detectors have been selected: the $91 \mathrm{~A}$ BEGe detector, that is reporting a very good energy resolution, the 91C BEGe detector, that shows a bad resolution, and the RG1 coaxial detectors.

To create DPLMS filters, pulses from ${ }^{228}$ Th calibration runs are used, the noise is evaluated during physics runs. The flattop condition and the pile-up removal are needed for proper energy reconstruction. DPLMS filters obtained for the 91C BEGe and the RG1 coaxial detectors are reported in Fig. 10.

The comparison with other filters is performed by fitting the $2.6 \mathrm{MeV}$ peak of the calibration spectra. The results are reported in Tab. I for gaussian, ZAC and DPLMS filters in the three selected GERDA detectors.

The energy resolution improves using the ZAC filter in comparison with the results of the gaussian filter. A further improvement is produced with the DPLMS filter: the FWHM improvement is in the range of $6-12 \%$ and $2-4 \%$ with respect to gaussian and ZAC filter respectively. 


\section{CONCLUSIONS AND OUTLOOK}

The adopted solution for the GERDA read-out electronics has been presented. A new read-out has been realized for the LEGEND-200 experiment, that will replace GERDA. The new design includes a separation in two stages: the goal is not to degrade the excellent performance of Ge detectors. First tests reported good results in terms of energy resolution, dynamic range, bandwidth and power consumption.

The particular GERDA condition requires a non-standard shaping filter, currently a modified cusp-like filter is used. To take into account a more general noise situation, we started the implementation of an optimum filter: our choice is the DPLMS filter. Promising preliminary results has been obtained in a first application to signal coming from GERDA detectors and a further improvement is expected with more specialization. In addition a future implementation of the DPLMS filter is planned to improve the PSD power, that is much more affected by high-frequency sinusoidal disturbances.

\section{ACKNOWLEDGMENT}

The GERDA experiment is supported by the German Federal Ministry for Education and Research (BMBF), the German Research Foundation (DFG) via the Excellence Cluster Universe, the Italian Istituto Nazionale di Fisica Nucleare (INFN), the Max Planck Society (MPG), the Polish National Science Centre (NCN), the Foundation for Polish Science (TEAM/20162/17), the Russian Foundation for Basic Research (RFBR) and the Swiss National Science Foundation (SNF). These research institutions acknowledge internal financial support. This project has received funding/support from the European Unions Horizon 2020 research and innovation programme under the Marie Sklodowska-Curie grant agreements No 690575 and No 674896. The GERDA Collaboration thanks the directors and the staff of the LNGS for their support of the GERDA experiment.

\section{REFERENCES}

[1] Gerda Collaboration, Eur. Phys. J. C 732330 (2013)

[2] GERDA Collaboration, Phys. Rev. Lett. 111, 122503 (2013)

[3] GERDA Collaboration, Eur. Phys. J 78388 (2018)

[4] Gerda Collaboration, Eur. Phys. J. C 7539 (2015)

[5] Janicskò-Csáthy J. et al., (arXiv:1606.04254)

[6] GERDA Collaboration, Nature 544, 7648 (2017)

[7] GERDA Collaboration, Phys. Rev. Lett. 120132503 (2018)

[8] Zsigmond A.J. for the GERDA Collaboration, Neutrino 2018, Heidelberg

[9] MAJORANA Collaboration, (arXiv:1902.02299)

[10] LEGEND Collaboration, AIP Conf. Proc. 1894, 020027 (2017)

[11] Kermaidic Y. et al., Nucl. Instrum. Meth. A 891, 106 (2018)

[12] Riboldi S. et al., ANIMMA 2015, Lisbon (Portugal), pp. 1-6

[13] D'Andrea V., PhD Thesis (2017)

[14] M. Willers, Private communication

[15] Gerda Collaboration, Eur. Phys. J. C 75255 (2015)

[16] Gatti E. et al., Nucl. Instr. Meth. Phys. Res. A 523 (2004) 167-185 